\title{
Tecnura
}

\section{Prototipo de cosecha inteligente de agua lluvia para mejorar la eficiencia energética residencial en Bogotá}

\section{Intelligent Rainwater Harvesting Prototype to Improve Residential Energy Efficiency in Bogota}

\author{
Carlos Arturo Ramírez-Escobar (iD) 1, Clara Inés Buriticá-Arboleda (iD)
}

Fecha de Recepción: 23 de Noviembre de 2020

Fecha de Aceptación: 01 de Abril de 2021

Cómo citar: Ramírez-Escobar., C.A. y Buriticá-Arboleda., C.I. (2021). Prototipo de cosecha inteligente de agua lluvia para mejorar la eficiencia energética residencial en Bogotá. Tecnura, 25(69), 171-195. https:/ / doi.org/10.14483/22487638.17975

\section{Resumen}

Objetivo: Presentar los resultados e impactos de un prototipo de cosecha inteligente de agua lluvia diseñado para la sustitución parcial de agua potable, incluyendo la energía embebida requerida para su producción y distribución, en una vivienda unifamiliar en Bogotá (Colombia).

Contexto: La ciudad de Bogotá, posee un régimen bimodal de precipitaciones frecuentes con mínimos mensuales multianuales que superan los $30 \mathrm{~mm}$ y como promedio superan los $60 \mathrm{~mm}$. Esta frecuencia de lluvias representa un potencial hídrico y energético, almacenado en la atmósfera, disponible localmente durante la mayor parte del año, de manera que posibilita su realización bajo esquemas novedosos de diseño tecnológico y económico de los sistemas de cosecha de aguas lluvias (SCALL). En este artículo nos centraremos en el establecimiento de las bases de diseño de un prototipo de cosecha de agua lluvia unifamiliar que logre capturar las potencialidades pluviales y energéticas de su entorno y pueda competir eficientemente frente al suministro de agua de la Empresa de Acueducto de Bogotá (EAB). Este permitirá sustituir los usos de agua potable en inodoros, lavado de ropa, riego de jardines y limpieza de espacios y tanques, lo cual se logrará con menor consumo de energía que el proveedor centralizado de agua potable.

Método: Se parte de considerar al agua lluvia como un recurso distribuido, tanto de agua como de energía, que tiene la capacidad de sustituir al agua potable provista como servicio energético de abastecimiento centralizado de agua de la EAB. Para evaluar la eficiencia de la sustitución de agua potable por agua lluvia cosechada, se genera un nuevo método que en solo cinco pasos permite evaluar la eficiencia comparativa, tomando como línea de base la proyección del desempeño del servicio centralizado de agua potable, respecto a la medida de mejoramiento del desempeño del prototipo SCALL. Para ello, se toma como principal indicador de desempeño a la intensidad energética comparada; la cual, a su vez, posibilita el cálculo de beneficios múltiples de la eficiencia energética.

Resultados: El prototipo SCALL, en las etapas iniciales de su implementación, logró ahorros del agua potable utilizada en la vivienda del $25 \%$, de la energía embebida del $26 \%$ y el abatimiento de los gases de efecto invernadero en un $27 \%$.

\footnotetext{
${ }^{1}$ Ingeniero mecánico, magíster en Turbinas, magíster en Ingeniería Eléctrica, doctor en Gestión Eficiente de la Energía Eléctrica. Profesor-investigador de la Universidad Autónoma de Colombia. Bogotá, Colombia. Email: ramirez.carlos@fuac.edu.co

${ }^{2}$ Ingeniera electricista, especialista en Informática Industrial, doctora en Gestión Eficiente de la Energía Eléctrica. Profesorainvestigadora de la Universidad Distrital Francisco José de Caldas. Bogotá, Colombia. Email: ciburiticaa@udistrital.edu.co
} 


\title{
Tecnura
}

Esto fue obtenido con apenas un 22,4\% de la capacidad nominal de diseño del prototipo, en un periodo de estudio de 56 meses. En este periodo se logró recuperar la inversión a partir de los ahorros económicos producto de la sustitución del agua potable por agua lluvia. Los resultados sugieren que la EAB podría desarrollar un programa de implementación de SCALL eficientes por parte de sus usuarios, lo que le permitiría obtener cerca de un ingresos y ahorros dinerarios que representarían cerca del $2 \%$ del total de ingresos que produciría cada SCALL residencial. El grado de éxito dependería de la masificación del programa. Esto sin contabilizar los beneficios que obtendría al aplazar las inversiones por ampliación de infraestructura, seguridad de abastecimiento y confiabilidad que proporciona la implementación de los SCALL eficientes. Conclusiones: Los resultados de la evaluación de la eficiencia energética arrojan la completa viabilidad tecnológica, económica, ambiental y social del prototipo SCALL de un usuario residencial en Bogotá. A la vez se dispone de una nueva metodología para la evaluación del potencial de eficiencia energética y sus beneficios múltiples cuando es escrutado un servicio energético provisto por un recurso distribuido como es el agua lluvia. Para el Distrito Capital de Bogotá resulta conveniente tanto social y ambientalmente desarrollar una política de promoción del SICCALL como medida de gestión eficiente de la demanda de agua y energía. Esta medida puede ser posibilitada por el desarrollo de economías de red y colaborativas, alrededor de recursos distribuidos de agua y energía, en la vía del cumplimiento de los Objetivos de Desarrollo Sostenible y participación ciudadana, de las llamadas ciudades inteligentes.

Palabras clave: cosecha de agua lluvia, eficiencia energética, intensidad energética, conservación del agua, respuesta de la demanda, servicios energéticos.

\begin{abstract}
Objective: To present the results and impacts of a prototype for harvesting rainwater in a single-family house in Bogota (Colombia), designed to save both potable water consumption as well as the embodied energy required to produce and distribute it from the city water system.

Context: The city of Bogota has a bimodal rainfall regime with multiannual monthly minima above $30 \mathrm{~mm}$, and an average above $60 \mathrm{~mm}$. This high rainfall intensity and frequency constitutes a potential hydric and energetic resource, which is available to the region during most of the year, and could be extracted through new systems for rainwater collection and use (referred to as SCALLs, for its acronym in Spanish). In this article, we will focus on the initial design of a rainwater harvester prototype with a capacity for one family. This prototype has been designed to operate under the high rainfall potential of the city, in order to store and deliver water efficiently to a household connected to the main local water provider (the Aqueduct Company of Bogota, EAB in Spanish). The water supplied by the prototype is enough to substitute the consumption of tap water for the usage of toilets, washing machine, watering of gardens, and cleaning of tanks and general surfaces. In comparison to the water provider, the rainwater harvester prototype is competitive with the quantity of water it delivers, and with the reduced consumption of energy required to supply it.
\end{abstract}

Method: To start the analysis of potential energy efficiency, we considered rainwater as a composition of two distributed resources: the water itself, and its embedded energy. Thus, rainwater has the potential to both replace the tap water consumption and reduce the energy service provided by the centralized water supply of the EAB. To evaluate the efficiency of this substitution, we generated a new 5-step method that allowed us to define the baseline efficiency as a performance projection of the centralized tap water service, and then compare it to the performance improvement of the SCALL prototype. We found that the main performance indicator is the comparative energy intensity, which enables the calculation of multiple benefits of energy efficiency.

Results: The SCALL prototype, in the initial stages of its implementation, achieved savings of $25 \%$ in drinking water used in the home, $26 \%$ of embedded energy and a reduction of greenhouse gases by $27 \%$. This was obtained with only $22.4 \%$ of the nominal design capacity of the prototype, in a study period of 56 months. In this period, it was possible to recover the investment from the economic savings resulting from the substitution of drinking water for rainwater. The results sug- 
gest that the EAB could develop an efficient SCALL implementation program by its users, which would allow it to obtain close to an income and monetary savings that would represent close to $2 \%$ of the total income that each residential SCALL would produce. The degree of success of which would depend on the massification of the program. This without accounting for the benefits that would be obtained by deferring investments for infrastructure expansion, security of supply and reliability provided by the implementation of efficient SCALLs.

Conclusions: The results of the energy efficiency evaluation show the technological, economic, environmental and social viability of the SCALL prototype of a residential user in Bogotá. At the same time, we propose a new methodology for evaluating the energy efficiency potential from a distributed resource such as rainwater, allowing scrutiny of its multiple benefits compared to a centralized service supplier. For the Capital District of Bogotá, it would be beneficial (socially and environmentally) to develop a policy to promote SICCALL to efficiently manage the demand for water and energy. This policy could be made possible by the development of collaborative network economies, around distributed water and energy resources, in order to meet the objectives of sustainable development and citizen participation, of the so-called smart cities.

Keywords: rainwater harvesting, energy efficiency, energy intensity, water conservation, demand response, energy services.

\section{Tabla de Contenidos}

Método

Elaboración de la línea de base . . . . . . . . . . . . . . . . . . . . . . . . . . 179

Desglose del pronóstico de referencia . . . . . . . . . . . . . . . . . . . . . 181

Caracterización de las medidas de eficiencia . . . . . . . . . . . . . . . . . . . . 181

Valoración de los resultados de beneficios múltiples . . . . . . . . . . . . . . . 185

Medición de los efectos económicos y otros efectos de recursos . . . . . . . . . . . . . . 186

$\begin{array}{ll}\text { Discusión de resultados } & 187\end{array}$

$\begin{array}{lr}\text { Conclusiones } & 190\end{array}$

$\begin{array}{ll}\text { References } & 191\end{array}$

\section{Introducción}

En las ciudades existen dos tipos de sistemas de cosechas de aguas lluvias (SCALL): a) el centralizado, el cual está asociado a las escorrentías por el suelo urbano y usualmente involucra a la infraestructura del sistema de drenaje citadino (alcantarillado de aguas pluviales) y puede abarcar el servicio comunitario, o del sistema de acueducto de la urbe; y b) el descentralizado, asociado al 
vertido de las cubiertas de los edificios y casas citadinas y que involucra infraestructura construida o modificada con tal finalidad (Retamal, Abeysuriya, Turner y White, 2009, p. 5).

En los edificios y casas citadinas, los SCALL son concebidos, diseñados e implementados para cumplir los fines del consumo industrial, de servicio o de vivienda y se especifican principalmente como bien sustituto en los usos finales, donde no es requerida la calidad potable del agua suministrada por el acueducto centralizado; aunque se encuentran aplicaciones donde el agua lluvia se plantea como sustituto económico del suministro de agua de toda la edificación.

Los sistemas residenciales de cosecha de agua lluvia pueden ser unifamiliares y/o comunitarios (Agatón, Ruiz y Sayago, 2016). En este artículo nos centraremos en el establecimiento de las bases de diseño de un prototipo de cosecha de agua lluvia unifamiliar que logre capturar las potencialidades pluviales y energéticas de su entorno, y pueda competir eficientemente frente al suministro de agua de la Empresa de Acueducto de Bogotá (EAB). Este permitirá sustituir los usos de agua potable en inodoros, lavado de ropa, riego de jardines y limpieza de espacios y tanques, lo cual se logrará con menor consumo de energía que el proveedor centralizado de agua potable.

La competencia por esta sustitución es especialmente exigente para los usuarios del servicio, ya que en la estructura tarifaria del servicio de suministro de agua, efectuado por la EAB, existen costos hundidos y otros beneficios no reconocidos, como los siguientes:

a. Se excluye, en la práctica, el costo del uso del agua (Lizcano, Congote y Angarita, 2012).

b. Otra distorsión del costo es abonable a la disminución del aforo contabilizado para el cobro tarifario del servicio de alcantarillado (a la salida de aguas servidas), cuando se utiliza un SCALL para fines residenciales, en virtud de la carencia de medición en la salida de la residencia.

c. En la estructura tarifaria no se tienen en cuenta los beneficios ambientales que trae consigo la retención y uso de agua lluvia, como el decremento de la huella hídrica de EAB (Pérez, Flores, González y Mota, 2019), así como el incremento de la huella verde del agua residencial al utilizar sistemas y recursos distribuidos de suministro de agua como los SCALL.

d. Tampoco se tienen en cuenta los atributos de costos del servicio energético que posee el suministro de agua en forma de energía embebida durante la prestación del servicio de acueducto y alcantarillado (Ürge-Vorsatz et al., 2012, pp. 665-669), cuya eficiencia podría ser transada en mercados meritorios de seguridad de abastecimiento eléctrico (Buriticá-Arboleda, RamírezEscobar y Álvarez-Bel, 2019), como participación activa de la demanda (Ramírez-Escobar, 2012, Ramírez-Escobar, 2007).

e. Así mismo, no se tienen en cuenta los costos evitados por emisión de gases de efecto invernadero (GEI), debido a la disminución de la huella de carbono al alcanzar mayor eficiencia energética durante la implantación de los SCALL. 
En estas condiciones, la viabilidad de la utilización del SCALL, en Bogotá, está mucho más ligada a los méritos de diseño e implementación que pueda exhibir, su propia estructura de costos de inversión, al ser comparada con los méritos de la estructura de costos del servicio centralizado de la EAB; institución que ha sido certificada en 2016 como empresa de carbono neutro (ICONTEC, 2016). En consecuencia, un usuario-responsivo que desee implementar su propio SCALL debe previamente considerar la gestión eficiente de su demanda de agua, basada en tres aspectos complementarios a saber:

a. La conservación de los recursos hídricos, que se adopta en los edificios mediante el uso de aparatos y accesorios eficientes en el uso del agua (Carragher, Stewart y Beal, 2012,Manco Silva, Guerrero Erazo y Ocampo Cruz, 2012,US EPA, 2016).

b. La información de los patrones y hábitos de consumo de agua (Stewart, Willis, Panuwatwanich y Sahin, 2013), ya que la reducción de la demanda de agua también depende de un cambio en el comportamiento del uso del agua (Acosta Alarcón, Rodríguez y Kua, 2019, Goette, Leong y Qian, 2019, Tijs et al., 2017), obtenida mediante tecnologías de visualización y campañas de sensibilización (García Lirios et al., 2013).

c. La implementación de las opciones de suministro de agua descentralizado a los edificios, como la sustitución de fuentes de agua potable con agua reciclada o lluvia (SCALL).

En consecuencia, es deseable que el SCALL forme parte y / o coadyuve a la creación de un sistema de administración y manejo del lado de la demanda del suministro de agua, pues es claro que las medidas de conservación y el cambio de patrones de uso aumentan la eficiencia del SCALL.

Se parte de la premisa de que los SCALL son amigables con el medio ambiente, y el agua lluvia es un recurso renovable; sin embargo, uno de los temas emergentes -surgido de los estudios del ciclo de vida en las ciudades que poseen programas de gestión de la demanda a través de los SCALL, al evaluar los nexos agua/energía- consiste en que su eficiencia energética comparativa con la de los acueductos centralizados, en la práctica, puede tener un pobre desempeño (Vieira et al., 2014). El indicador mayormente utilizado para evaluar esta comparación es la intensidad energética (es decir, unidad de energía por unidad de agua), pues permite juzgar la viabilidad ambiental de los SCALL, frente a la evaluación del ciclo de vida (o una de sus fases, suministro y/o servicios de aguas residuales). Este hallazgo contraevidente expuesto en (Vieira et al., 2014, p. 23) se muestra en la figura 1 .

(Vieira et al., 2014) afirman que, en los estudios teóricos revisados, la mediana de la intensidad energética $\left(0,20 \mathrm{kWh} / \mathrm{m}^{3}\right)$ fue considerablemente menor que la descrita en los estudios empíricos $\left(1,40 \mathrm{kWh} / \mathrm{m}^{3}\right)$, debido a que las evaluaciones teóricas de la intensidad energética no pueden considerar suficientemente la energía utilizada para el arranque de la bomba y el modo de espera, así como la verdadera eficiencia energética del motor y la bomba. Al comparar los SCALL con los sistemas convencionales de suministro de agua de la ciudad, los estudios empíricos revisados mostraron 


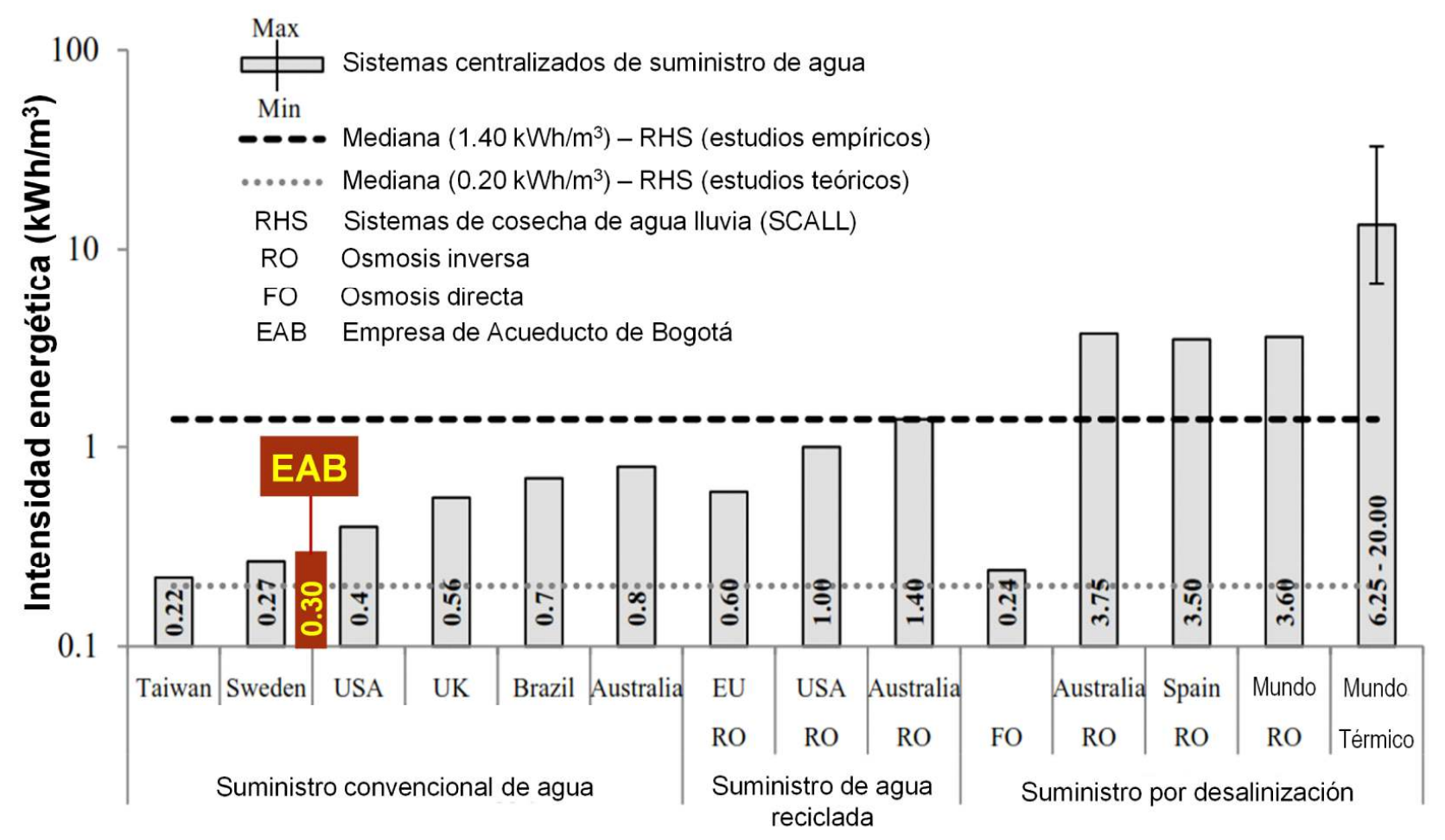

Figura 1. Comparación de la intensidad energética entre los sistemas de suministro centralizados y los SCALL (RHS)

Fuente: adaptado de (Vieira et al., 2014, p. 23).

que los SCALL tienden a consumir tres veces más energía.

Los estudios teóricos, experimentales y empíricos han proporcionado pistas importantes para mejorar la eficiencia energética de los SCALL, que incluyen:

a. El uso de tanques de cabecera para mejorar el rendimiento de los sistemas de bombeo al reducir el número de arranques de la bomba y ajustar el caudal al mejor punto de eficiencia (Sharma, Begbie y Gardner, 2015, pp. 127-149).

b. El uso de bombas de baja presión para reducir la energía total incorporada al agua de lluvia (Tjandraatmadja, Retamal, Umapathi y Hauber-Davidson, 2015).

c. El uso de diámetros de tubería más grandes para reducir las pérdidas por fricción. 
d. El uso de suministro directo de agua de lluvia con almacenamiento de agua de lluvia en tanques de cabecera y distribución por gravedad sin bombeo (Angrill et al., 2012).

e. El aumento de la demanda de agua de lluvia para reducir la intensidad energética asociada con el modo de espera y el arranque de la bomba.

f. Para edificios de varios pisos, los arreglos de sistemas multitanques en cascada de cosecha de agua lluvia (SMTCCALL) que disminuyen la cantidad de volumen de agua de necesario bombeo, al alimentar los tanques y suministrar por gravedad a los distintos usos (Sendanayake, 2010).

Sin embargo, en última instancia, son las características locales -como la oferta y la demanda de agua de lluvia, el tipo de edificio (de una sola planta o de varias plantas), el diseño de subsistemas SCALL, el diseño del sistema de plomería de agua potable, la intensidad de la energía del agua de la ciudad, entre otros factores- las que determinarán si los rendimientos ambientales y económicos de los SCALL son aceptables. Es decir, el mejoramiento de la eficiencia de los SCALL sigue siendo un traje diseñado a la medida del usuario.

Por tal razón, es menester analizar las características locales que son determinantes para la valoración de potenciales de eficiencias energéticas y de ahorro de agua; así, entonces, la oferta de agua lluvia en Bogotá está sujeta en gran medida a los determinantes climáticos del bosque tropical andino contiguo a la selva del Amazonas, la cual determina las cantidades de lluvia conjuntamente con los fenómenos que produce la Oscilación Sur (ENSO) de El Niño y La Niña, pues, la selva del amazonas produce el efecto de bomba biótica (Makarieva y Gorshkov, 2007, Makarieva, Gorshkov y Li, 2009). En esta, por su evotranspiración, la cuenca selvática produce un río ascendente, mucho más grande en caudal, que el de su escorrentía en el suelo. La succión de esta bomba biótica está orientada a la masa de aire húmedo del océano Atlántico, que trae consigo el polvo del desierto del Sahara, rico en fósforo, que fertiliza la masa biótica amazónica, como lo evidencia la investigación espacial de la (ScienceAtNASA, 2015).

Este fenómeno de la bomba biótica incide directamente sobre las precipitaciones de la cuenca oriental de los Andes colombianos y del piedemonte llanero, como afirman (Bunyard y Herrera, 2012). En consecuencia, la ciudad de Bogotá posee un régimen bimodal de precipitaciones frecuentes con mínimos mensuales multianuales que superan los $30 \mathrm{~mm}$ y como promedio superan los $60 \mathrm{~mm}$ (Climate-Data.org, 2019). El aprovechamiento de esta bomba biótica amazónica determina al diseño dimensional y energético de los SCALL de la ciudad de Bogotá y sus zonas aledañas, alejándolo de los parámetros y disposiciones convencionales previstos en los diseños de otras latitudes; pues, como producto de esta bomba biótica se puede obtener caudal de agua y cabeza hidráulica (energía) disponible en la atmósfera durante gran parte del año. Este potencial puede ser aprovechado de mejor manera por los sistemas de cosecha de agua lluvia multitanque en cascada, alimentados por gravedad. Estos sistemas podrían coadyuvar a intensificar el nexo agua/energía en las zonas rurales 
propias y circundantes a Bogotá, proveyendo con alta eficiencia el recurso hídriconecesario de los biodigestores residenciales rurales de las fincas agropecuarias de pequeña escala (Ladino Tamayo, Martínez Rojas y Buriticá-Arboleda, 2018).

Todo este panorama justifica el desarrollo de fuentes alternativas distribuidas de provisión de agua, en especial de agua lluvia que desplace el uso de agua potable donde no sea necesaria, sobre todo en los ámbitos urbanos y de ruralidad colindante a la ciudad. Para ello, este artículo propone el desarrollo de una tecnología para la cosecha eficiente e inteligente de agua lluvia en condiciones urbanas de la ciudad de Bogotá, a través de un prototipo denominado sistema inteligente en cascada para la cosecha de agua lluvia (SICCALL).

\section{Método}

El enfoque tradicional del ahorro de energía o de insumos, para las variadas actividades de los diferentes sectores de la producción y demanda de bienes y servicios, puede conducir a subestimar el valor total de la eficiencia en economías individuales o sectoriales, locales, regionales, nacionales e internacionales. Por ello, para el presente trabajo se parte conceptualmente de considerar al agua lluvia como un recurso distribuido, tanto de agua como de energía. Este se realiza dentro de una cadena de suministro como un servicio energético, susceptible de autoprovisión del lado de la demanda (Buriticá-Arboleda et al., 2020, p. 63); bajo este concepto se adapta el enfoque ascendente (bottom-up), contenido en la metodología analítica, para estudios de potencial de eficiencia energética, utilizada en el Plan de Acción Nacional para la Eficiencia Energética (NAPEE por sus siglas en inglés) en los Estados Unidos y preparada por (Mosenthal \& Loiter, 2007), y se integra con el enfoque de captura de múltiples beneficios de la eficiencia energética, de la Agencia Internacional de Energía (IEA, 2014).

La adaptación mencionada se centra en tres aspectos: a) trabajar la eficiencia del lado de la demanda y no de la oferta, manifiesta en decisiones y medidas de repuesta de la demanda al mercado de bienes y servicios; b) prever los ahorros de agua potable y su sustitución por agua lluvia; y c) prever los ahorros de energía embebida del servicio centralizado de acueducto a través de su sustitución por la energía que porta el agua lluvia y su correspondiente abatimiento de GEI.

De otra parte, para la aplicación de la metodología de potenciales de eficiencia, se prevé comenzar con los ahorros y costos asociados al mejoramiento en el uso del agua potable a nivel residencial; partiendo del cambio de hábitos, luego el cambio tecnológico y, por último, la innovación con un sistema multitanque en cascada, para la cosecha inteligente de agua lluvia. Este es el denominado enfoque ascendente, recomendado para los análisis residenciales, dada la disponibilidad de información y mayor homogeneidad de infraestructura e inventario de equipos a los que se aplican las medidas. El otro enfoque que se integra, el de la captura de múltiples beneficios de la eficiencia, acerca más a comprender el valor real de la eficiencia como recurso clave para el desarrollo económico y 
Tabla 1. Datos del usuario

\begin{tabular}{|l|c|c|c|c|c|}
\hline \multicolumn{2}{|c|}{ Inmueble } & \multicolumn{2}{c|}{ Suba } & (correspondencia) & Batan \\
\hline \multicolumn{2}{|c|}{ Estrato: } & 5 & \multicolumn{2}{c|}{ Clase de uso: } & Residencial \\
\hline Unidad habitacional familiar: & 1 & Unidad no habitacional: & 0 \\
\hline Zona: & 1 & Ciclo: & Q1 & Ruta: & Q11626 \\
\hline \multicolumn{7}{|c|}{ Datos del medidor } \\
\hline Marca: & Watertech Italia & Tipo: & VELO015T2 & Diámetro: & \multicolumn{2}{c|}{${ }^{1 / 2}$} \\
\hline \multicolumn{2}{|c|}{ Facturado con: } & \multicolumn{2}{|c|}{ Consumo normal } & Descargue fuente alterna & 0 \\
\hline
\end{tabular}

Fuente: elaboración propia.

social (IEA, 2014), que va más allá de la evaluación económica y financiera de un proyecto privado de inversión, al identificar y medir los impactos de la eficiencia energética en múltiples aspectos y descubrir muchos beneficios del nexo bidireccional agua/energía.

Por tanto, la metodología planteada para estudiar los impactos del prototipo de cosecha inteligente de agua lluvia, en la mejora de la eficiencia energética en una vivienda unifamiliar de Bogotá, adaptada de (Mosenthal \& Loiter, 2007), e (IEA, 2014), divide el proceso en cinco pasos, como se detallan a continuación:

\section{Elaboración de la línea de base}

Consiste en identificar el pronóstico del consumo de agua potable y su energía embebida, en la vivienda unifamiliar, que se tomará como referencia para evaluar los ahorros y mejora en la eficiencia energética; incluyendo una comprensión específica de lo que es y lo que no incluye, en términos de cambios futuros a códigos y estándares, programas de eficiencia planificados, entre otros. La vivienda unifamiliar se ubica en Bogotá, con altitud promedio por encima de $2000 \mathrm{~m} \mathrm{~s}$. n. m., lo que le regula un nivel de consumo básico de $11 \mathrm{~m}^{3}$ /suscriptor/mes. La tabla 1 muestra los datos del usuario, según la factura de la EAB.

En la unidad habitacional familiar residen 4 personas, es de 2 plantas con altura máxima de $10 \mathrm{~m}$, tiene aproximadamente 50 años de construida y cuenta con los servicios de acueducto y alcantarillado estándares para su tipo.

- Información histórica del consumo de agua potable. Se cuenta con el registro histórico de 2 años de la facturación bimensual del servicio de acueducto y alcantarillado, proporcionado por la EAB, desde enero de 2012 a diciembre de 2014; tal como se muestra en la figura 2.

- Pronóstico del consumo de agua potable como línea de base o de referencia. El pronóstico, como una forma de prever o predecir los consumos futuros de agua potableen la vivienda unifamiliar, se 


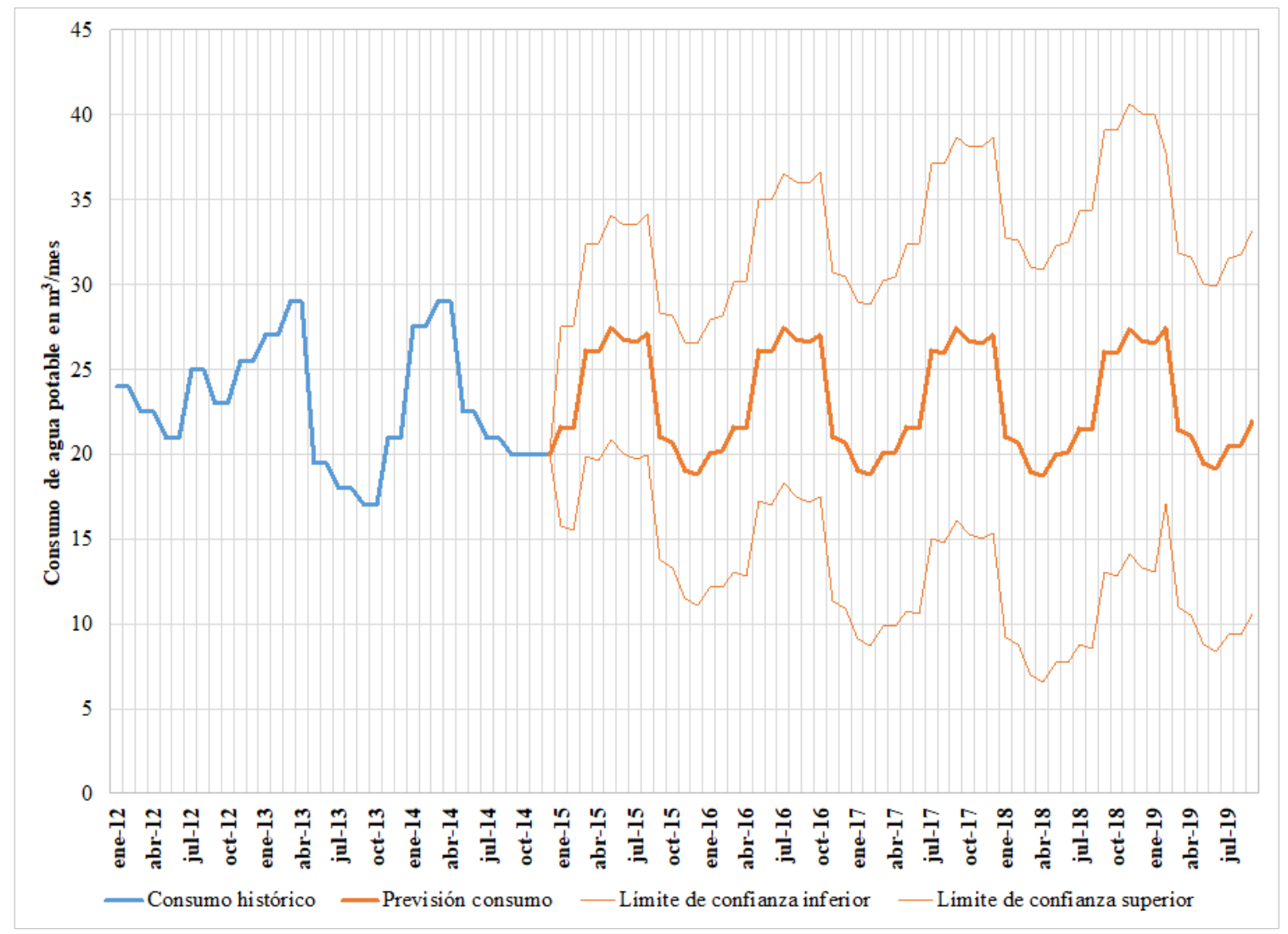

Figura 2. Línea base del consumo de agua potable en la vivienda unifamiliar de Bogotá

Fuente: elaboración propia.

elabora a partir de la información histórica y de algunas consideraciones adicionales en torno a la llamada eficiencia natural, que agrega a los pronósticos algunos ahorros de agua potable, con el fin de tener una línea de base más adecuada. La eficiencia natural, entendida como las acciones y medidas que influyen en los ahorros en ausencia de incentivos programáticos (Mosenthal \& Loiter, 2007), es una realidad en usuarios finales que, con los recursos disponibles, reaccionan a tarifas, precios, problemas ambientales, calidad de los servicios; como un mecanismo de respuesta de la demanda al sector de bienes y servicios, especialmente a los de servicios públicos domiciliarios.

En la figura 2, puede observarse la proyección de los consumos de agua potable de la vivienda unifamiliar, elaborada para el periodo comprendido entre enero de 2015 y enero de 2019. En la eficiencia natural se incluyeron medidas como: cambio de hábitos y costumbres, en el uso final del agua potable (como cambio a riego por goteo, disminución del tiempo de ducha, entre otros) y de otros recursos energéticos y la reposición natural de tuberías y dispositivos, obsoletos e ineficientes, como tuberías de hierro, inodoros y lavamanos. Se destaca la evidente 
estacionalidad en el consumo de agua potable, que refleja circunstancias particulares periódicas, procesos y situaciones cíclicas, de la actividad cotidiana de los residentes en la unidad habitacional.

- Pronóstico de energía embebida y su huella de carbono. El pronóstico de energía embebida del agua potable suministrada a la vivienda se calcula a partir de información suministrada por la EAB; para ello se adopta el valor del año 2014 de intensidad energética y de intensidad de emisiones como constante para los años inferiores a este, y el valor del año 2015 para los años superiores (tabla 2). Con la proyección de agua potable y estos indicadores se calculan los valores de la energía embebida y su impacto de emisiones de GEI, que se constituyen en la línea de base para calcular los ahorros energéticos y de mitigación de emisiones de GEI, debidos a la implementación del prototipo SICCALL.

Tabla 2. Indicadores de intensidad energética y de emisiones de GEI-EAB

\begin{tabular}{|c|c|c|}
\hline Descripción & $\mathbf{2 0 1 4}$ & $\mathbf{2 0 1 5}$ \\
\hline Consumo de energía eléctrica de la red en $\mathrm{kWh}$ & 170979467 & 149097178 \\
\hline Agua potable producida/año en $\mathrm{m}^{3}$ & 495480302 & 495863171 \\
\hline Intensidad energética en $\mathrm{kWh} / \mathrm{m}^{3}$ & 0,345 & 0,301 \\
\hline Intensidad de emisiones de GEI en $\mathrm{kg} \mathrm{CO} \mathrm{CO}^{2} / \mathrm{m}^{3}$ & 0,078 & 0,073 \\
\hline de agua potable producida & & \\
\hline
\end{tabular}

Fuente: elaboración propia a partir de (EAB, 2014, EAB, 2015).

\section{Desglose del pronóstico de referencia}

Este aplica para otros usos finales y en bienes y servicios sustitutos, apropiados para el análisis. En la tabla 3, columna 2, se relaciona el desglose del consumo de agua potable en la vivienda multifamiliar, en usos finales; en la columna 3, se indican aquellos usos que pueden ser sustituidos por agua lluvia.

Por tanto, se estima que un $56 \%$ del consumo de agua potable suministrada por la EAB puede ser sustituido por el agua lluvia cosechada a partir del prototipo SICCALL.

\section{Caracterización de las medidas de eficiencia}

Para este proyecto se prevé la sustitución de hasta un $56 \%$ del consumo de agua potable proyectado, por la cosecha inteligente de agua lluvia, descrito a continuación como el prototipo SICCALL (figura 3). 
Tabla 3. Usos del agua potable en el consumo total, en la vivienda multifamiliar

\begin{tabular}{|c|c|c|}
\hline Uso & \% uso final agua potable & \% a sustituir con agua lluvia \\
\hline Lavado de ropa & 27,1 & 27,1 \\
\hline Ducha & 20,9 & - \\
\hline Sanitarios & 19,9 & 19,9 \\
\hline Lavaplatos & 15,5 & - \\
\hline Aseo de vivienda & 4,9 & 4,9 \\
\hline Consumo propio & 3,9 & - \\
\hline Lavamanos & 3,7 & - \\
\hline Riego de jardines & 1,9 & 1,9 \\
\hline Lavado de auto & 1,5 & 1,5 \\
\hline Riego de plantas & 0,7 & 0,7 \\
\hline Total & 100 & 56 \\
\hline
\end{tabular}

Fuente: elaboración propia a partir de (Junca Salas, 1999).

a. El prototipo SICCALL fue diseñado para las características de una vivienda existente, respondiendo con sus particularidades a las oportunidades y restricciones. Aunque es un traje a la medida, tiene la propiedad de ser generalizable, replicable y escalable, en virtud de sus principios físicos y energéticos funcionales, de carácter universal. Sin embargo, sus atributos de máxima eficiencia fueron optimizados para las condiciones de pluviosidad bimodal de Bogotá.

b. El principio universal que rige el diseño del prototipo SICCALL es la ley de conservación de energía que toma expresión particular en los fluidos como las leyes de Bernoulli y la de continuidad. La aplicación de la primera ley determina las alturas energéticas potenciales y dinámicas del fluido, que permiten diseñar el sistema multitanques, para proveer presión y volumen adecuados para los distintos usos del agua lluvia. La ley de continuidad del fluido permite diseñar las conexiones y depósitos reduciendo las pérdidas de manera que el sistema pueda ser energoeficiente. De esta manera se puede proveer, por gravedad, hasta un $45 \%$ del uso total de agua en la vivienda objeto de este prototipo.

c. El prototipo SICCALL está compuesto de los siguientes sistemas funcionales (figura 3):

- Acopio y captación de agua lluvia. Organiza la oferta de agua lluvia disponible en la residencia, para asegurar el abastecimiento en términos de calidad del agua, presión y continuidad (volumen en el tiempo) a un costo sostenible. Este sistema dispone de los subsistemas 


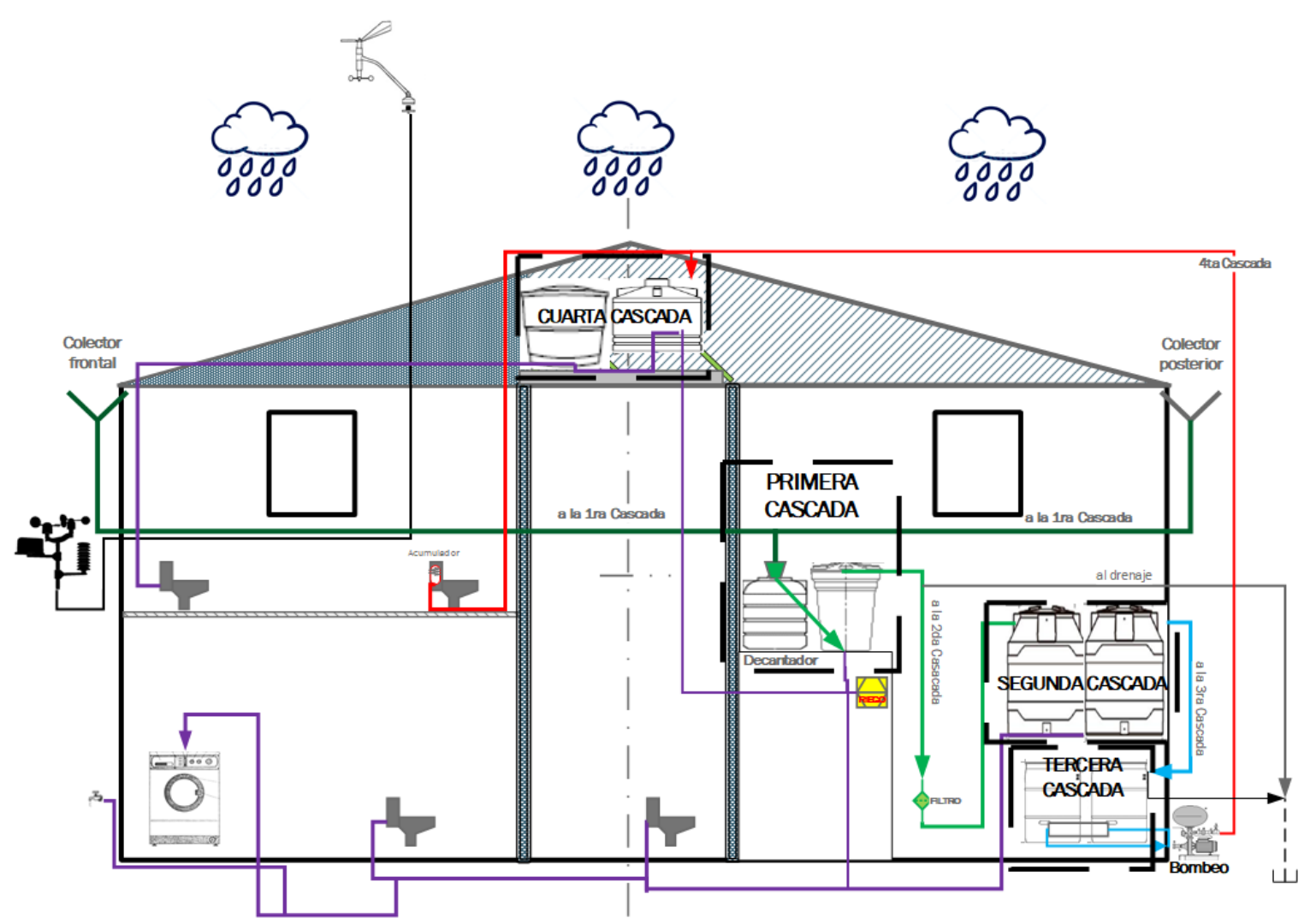

Figura 3. Esquema del prototipo sistema inteligente en cascada para la cosecha de agua lluvia (SICCALL)

Fuente: elaboración propia.

de captación, filtración y almacenamiento de agua decantada.

- Almacenamiento de agua lluvia. Almacena el agua lluvia filtrada para diferir en el tiempo los caudales y volúmenes almacenados, llevándolos y concentrándolos en un sistema multitanques de 4 cascadas, cuya operación se rige por el algoritmo de desempeño yield after spillage (YAS), el cual es un modelo conductual genérico para los sistemas de cosecha de agua lluvia, cuyas variables son: la demanda anual (D), la capacidad de almacenamiento (S), el área colectora (A) y el promedio anual de lluvia (R); en ecuaciones que son formuladas para determinar la cantidad de la lluvia colectada que debe ser bombeada a cada piso o a cada tanque en su respectivo nivel, logrando un desempeño de sistema dinámico para eficiencia energética del agua cosechada (Sendanayake, 2016). La cuarta cascada la constituye un sistema presurizado de suministro al tanque de cabecera y al inodoro de acumulación de presión y bajo consumo de agua (ver figura 3).

- Distribución para usos convencionales. Su función es transportar y distribuir el agua lluvia a los puntos de consumo, en la calidad, volumen y presión especificados con la mayor continuidad posible, a costos razonables. 
- Extracción de aguas residuales. Su función es recolectar y extraer los caudales de aguas grises o negras y excedentarias de lluvias que sean desalojadas por los desagües de cada equipo o dispositivo utilizado.

- Sistema de supervisión, control y vigilancia. Se compone de tres subsistemas: el de monitoreo climático, compuesto por una estación meteorológica con capacidad predictiva de lluvias; el de control electromecánico, que controla el encendido de la bomba en función de los niveles de los tanques de la tercera y cuarta cascada; y el inteligente de control, que consta de procesamiento continuo de datos de sensores y actuadores para la toma de decisiones de gestión del prototipo.

d. La operación del SICCALL prevé la recogida del agua lluvia mediante dos colectores (canales) adosados al techo de la vivienda, que conducen a sendas tuberías a través de la primera cascada a un filtro de autolavado y un decantador de doble volumen, los cuales recogen las impurezas gruesas del agua lluvia. Posteriormente, por rebose en el decantador, el agua lluvia se dirige a un tanque de almacenamiento de la primera cascada; este, por gravedad, alimenta los servicios ubicados en el primer piso de la vivienda. El agua rebosante de este tanque se dirige a un filtro de partículas finas conformado por carbón activado, grava y arena, que se encarga de la limpieza final del agua lluvia; y por gravedad, la lluvia es conducida a los tanques de almacenamiento de la segunda cascada, los cuales están diseñados para ser el principal receptáculo de almacenamiento del sistema $\left(1,2 \mathrm{~m}^{3}\right)$.

Estos tanques alimentan, por gravedad, a la carga de dos inodoros, al servicio de lavado de ropa, jardines y áreas de limpieza, ubicadas en el primer piso de la vivienda. El rebose de los tanques de la segunda cascada dirige el agua hasta los tanques inferiores de la tercera cascada, los cuales son el sistema de alimentación del sistema hidroneumático de bombeo. Este sistema está conformado por una bomba de $0,5 \mathrm{hp}$, con capacidad para alimentar a los tanques de la cuarta cascada con un caudal de $35 \mathrm{~L} / \mathrm{s}$, ubicados a $8 \mathrm{~m}$ de altura, en el entretecho de la vivienda.

Dicha bomba operada aisladamente del balón hidroneumático eleva $1 \mathrm{~m}^{3}$ en $28 \mathrm{~min}$ de operación, lo cual da como resultado una intensidad energética teórica de $0,071 \mathrm{kWh} / \mathrm{m}^{3}$, que correspondientemente, al consumir energía de la red eléctrica, tendría una intensidad teórica de emisiones de GEI de $0,015 \mathrm{~kg} \mathrm{CO}{ }^{2} \mathrm{e} / \mathrm{m}^{3}$, estos valores están considerados para el caso extremo de operación del sistema; que se presenta cuando el tanque hidroneumático no está disponible y el tanque de cabecera debe suplir las necesidades de la vivienda. La cuarta cascada alimentada por el sistema hidroneumático, que opera a presiones entre 26 y 40 psi, suministra agua lluvia a dos tanques y a un acumulador de presión inserto en uno de los inodoros eficientes del segundo piso de la vivienda. El segundo inodoro es alimentado por gravedad, por los tanques de cuarta cascada. Además, estos tanques operan como tanques de cabecera cuando la reserva de agua se agota para el primer piso. 
Tabla 4. Etapas e hitos del desarrollo del prototipo SICCALL

\begin{tabular}{|c|c|c|c|c|c|}
\hline Etapa & Hitos & $\begin{array}{c}\text { Fecha de } \\
\text { inicio y final }\end{array}$ & $\begin{array}{c}\text { Intensidad } \\
\text { energética } k W h / \mathbf{m}^{3}\end{array}$ & $\begin{array}{l}\text { Uso de } \\
\text { bombeo }\end{array}$ & $\begin{array}{c}\text { Volumen } \\
\text { instalado } \mathbf{m}^{3}\end{array}$ \\
\hline Et.I & $\begin{array}{l}\text { Instlación primera } \\
\text { cascada }\end{array}$ & $\begin{array}{c}\text { de enero } 2015 \\
\text { a dic } 2018\end{array}$ & 0 & No & 0,5 \\
\hline Et.II & $\begin{array}{l}\text { Instalación tercera } \\
\text { y (cuarta cascada); } \\
\text { y sistema control } \\
\text { electromecánico }\end{array}$ & $\begin{array}{l}\text { de enero } 2019 \\
\text { a agosto } 2019\end{array}$ & 0,07 & $\mathrm{Si}$ & 0,4 y $(0,25)$ \\
\hline Et.III & $\begin{array}{l}\text { Instalación segunda } \\
\text { cascada y (ampliación } \\
\text { cuarta cascada) }\end{array}$ & $\begin{array}{c}\text { de septiembre } \\
2019 \text { en adelante }\end{array}$ & 0,07 & $\begin{array}{l}\text { Parcial, } \\
\text { solo para } \\
\text { tanque de } \\
\text { cabecera }\end{array}$ & $1,2 \mathrm{y}(0,35)$ \\
\hline \multicolumn{5}{|c|}{ Capacidad del SICCALL en volumen total instalado } & 2,7 \\
\hline
\end{tabular}

Fuente: elaboración propia.

\section{Valoración de los resultados de beneficios múltiples}

Esta es pertinente, ya sea que dichos resultados sean directos o indirectos, por la implementación del prototipo SICCALL. Para entender esta valoración es necesario especificar los hitos y etapas del desarrollo, que el prototipo ha experimentado hasta la fecha (tabla 4).

Los resultados obtenidos de la implementación del prototipo SICCALL, en sus etapas de desarrollo, se muestran en la figura 4, para el ahorro concerniente al consumo de agua potable; además, allí se evidencian situaciones de no provisión de agua lluvia, debido a la conjunción de eventos de estrés climático ocurridos en el fenómeno de El Niño del 2015-2016, y la falta de capacidad de almacenamiento del prototipo, en su etapa inicial.

La evolución del ahorro de la energía embebida en el agua potable suministrada por la EAB (figura 5), durante la primera etapa de desarrollo del prototipo, es prácticamente similar al ahorro del consumo de agua potable, debido a que el suministro de agua lluvia provisto por el prototipo se efectuó exclusivamente a gravedad (sin bombeo). El desempeño de la segunda etapa a partir del inicio de 2019 operó el bombeo hacia el tanque de cabecera, el cual suministró preferentemente los servicios a los usos desplazados de agua potable. En consecuencia, es evidente la diferencia de áreas de ahorro (ralladas) entre el consumo de agua potable y la energía embebida, a partir de diciembre de 2018. 


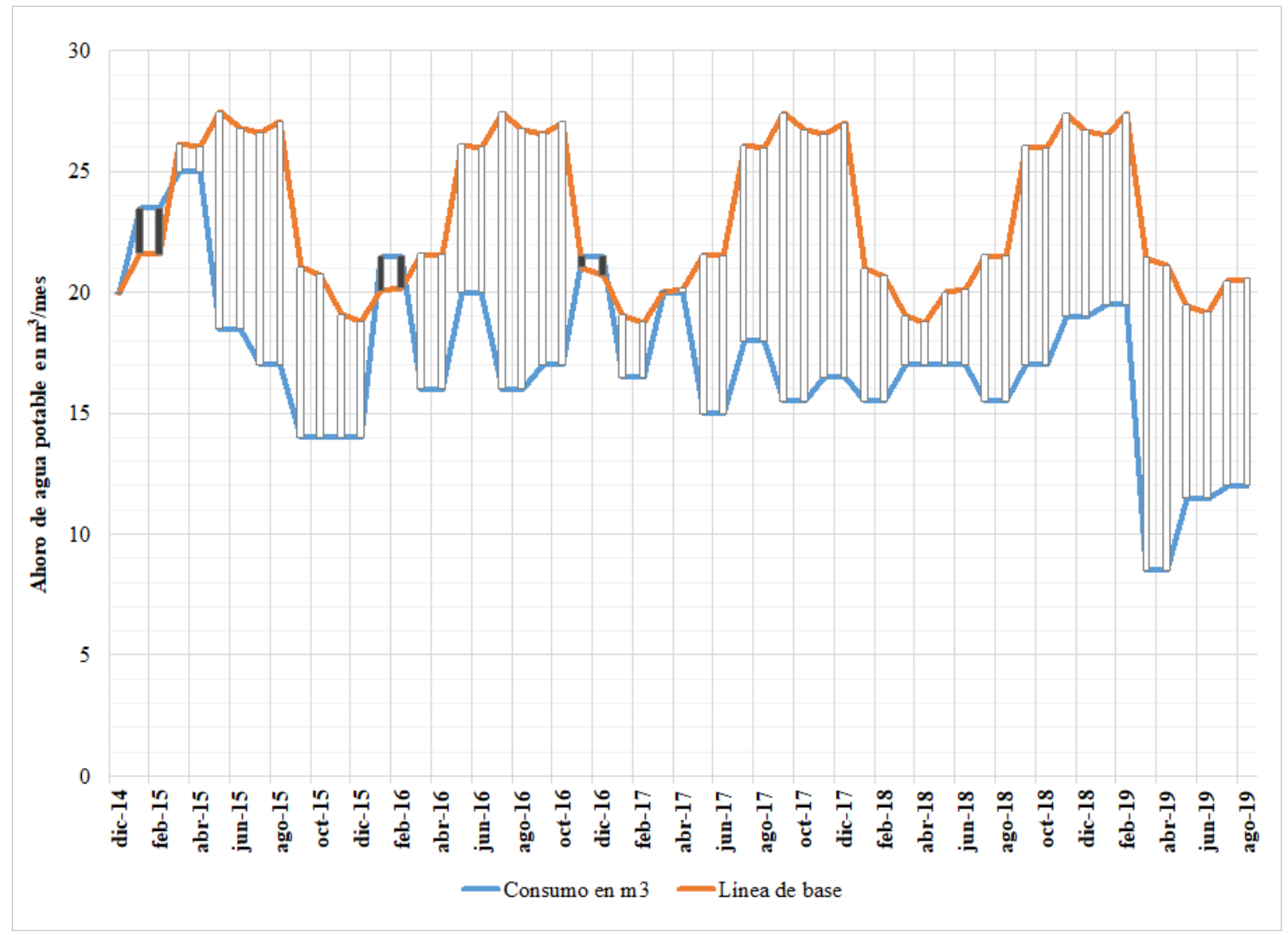

Figura 4. Evolución de los ahorros en el consumo de agua potable

Fuente: elaboración propia.

\section{Medición de los efectos económicos y otros efectos de recursos}

Los efectos económicos de la implementación del prototipo SICCALL parten del ahorro del agua potable y sus implicaciones, durante la explotación del prototipo en sus primeras fases, que se muestra en la figura 6. El ahorro en las primeras etapas de implementación del prototipo manifestó estacionalidad congruente con las condiciones de pluviosidad, puesto que reprodujo la escasez del fenómeno de El Niño 2015-2016; esto se explica en que el volumen útil del acopio de agua no fue suficiente para diferir en el tiempo su servicio.

En la figura 7 se muestra el costo unitario del servicio de agua potable de la EAB, el cual varía en el periodo de los cinco años estudiados, en aproximadamente un $25 \%$. Esta tarifa se convierte a su vez, en la de sustitución del agua potable por agua lluvia, la cual determinará en última instancia la relación costo/beneficio, ya que dicha tarifa define los ingresos, al afectar los flujos de ahorro.

La tabla 5 recoge los resultados totales de la evaluación del prototipo, durante el periodo de estudio 2015-2019 (56 meses), donde se obtuvo promedio mensual de sustitución de agua potable de $6 \mathrm{~m}^{3}$, lo que representa aproximadamente el $50 \%$ del potencial de diseño del prototipo SICCALL. 


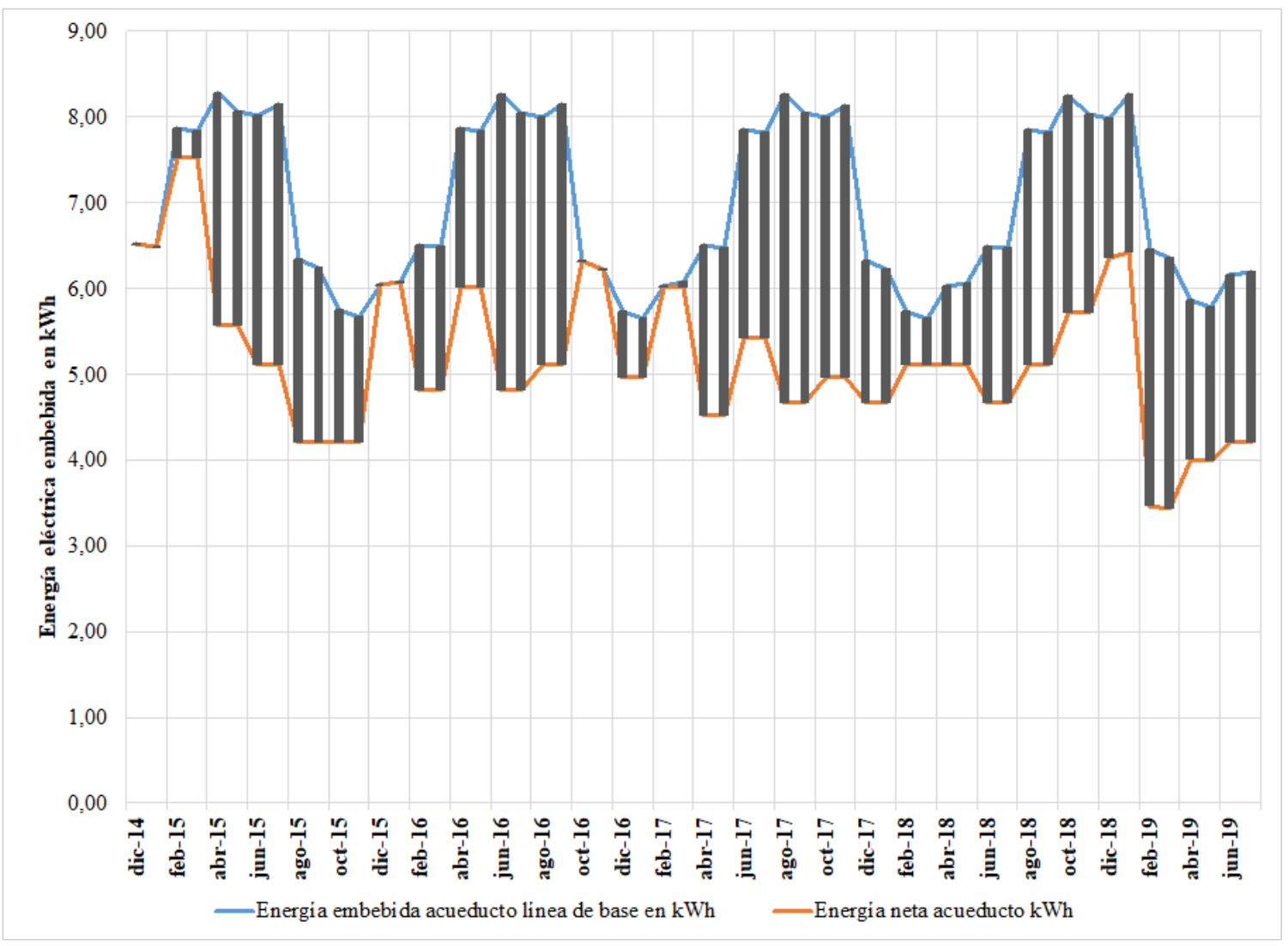

Figura 5. Evolución del ahorro de la energía embebida en el agua potable, suministrada por la Empresa de Acueducto de Bogotá (EAB)

Fuente: elaboración propia.

Sin embargo, permite observar la interdependencia entre las cantidades relativas de agua potable ahorrada (26\%), energía embebida ahorrada (25\%) y abatimiento de GEI en un $27 \%$, con respecto de la línea de base.

Los valores dinerarios de ingreso para cada uno de estos productos de la eficiencia hídrica y energética fueron calculados para las partes interesadas de usuario/propietario del prototipo y EAB, entidad promotora de programas de ahorro de agua y gestora de la demanda agregada.

\section{Discusión de resultados}

El periodo de análisis de este estudio, que comprende las etapas I y II (48 y 7 meses, respectivamente), arroja resultados que impactan los indicadores principales de eficiencia de consumo de agua potable, en $26 \%$; de disminución de la energía embebida en el agua potable, en $25 \%$, y por ende, de abatimiento de emisiones de GEI, en $27 \%$. Es destacable que estos indicadores de eficiencia se logran 


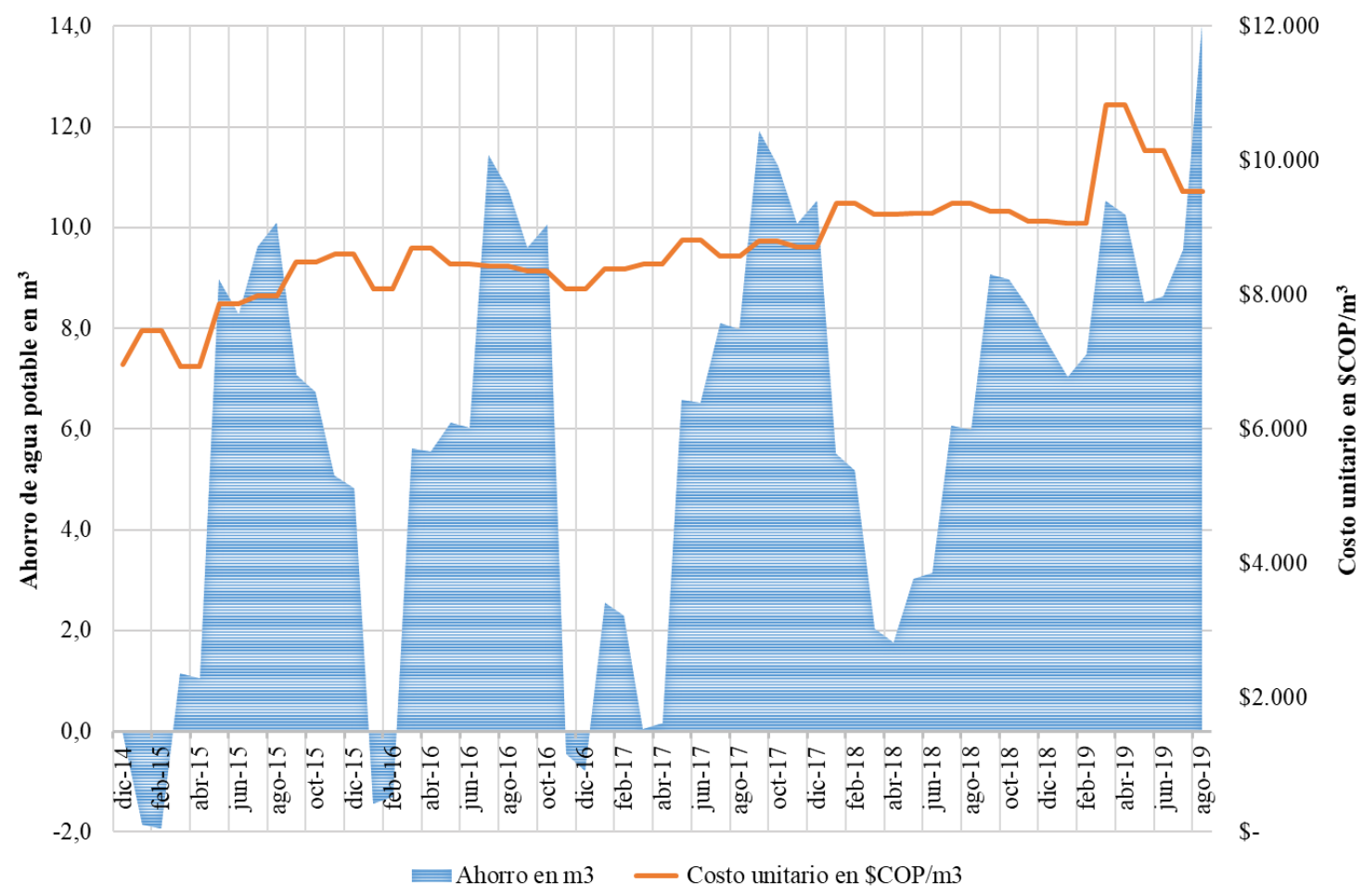

Figura 6. Evolución del ahorro de agua potable, a partir del prototipo sistema inteligente en cascada para la cosecha de agua lluvia (SICCALL)

Fuente: elaboración propia.

con un factor de instalación del volumen nominal del sistema del 19\% (etapa I), el cual sube a $46 \%$ en la etapa II; que en promedio ponderado equivale a $22,4 \%$. Es de esperar que cuando el prototipo logre su pleno volumen nominal y con la instalación del sistema de control inteligente, aumente ostensiblemente los indicadores de eficiencia descritos anteriormente. Este resultado es de gran importancia para el usuario, la EAB y el regulador, pues son una forma sostenible de lograr elevar las eficiencias y abatir emisiones de GEI; así como su incidencia en las futuras ampliaciones del sistema de suministro de agua para la ciudad.

Los valores absolutos de ahorro del volumen de agua potable -calculados en $338,4 \mathrm{~m}^{3}$, abatimiento de GEI de $25 \mathrm{~kg}$ y de ahorro de la energía embebida en el agua potable de $99 \mathrm{kWh}$ - no son aún muy dicientes de su verdadero potencial, ya que el prototipo SICCALL se desempeñó con un factor de instalación solo de 22,4\% de su capacidad nominal, durante el periodo de este estudio.

Los ingresos dinerarios, redituables al prototipo SICCALL mostrados en la tabla 5, por un monto total de COP 3084298 en el periodo de estudio de 56 meses, podrían tener una distribución según la parte interesada de la siguiente manera hipotética: a) el usuario/propietario recibiría el 98,3\% por concepto de la sustitución de agua potable, lo que le permitiría el retorno de la inversión durante el 


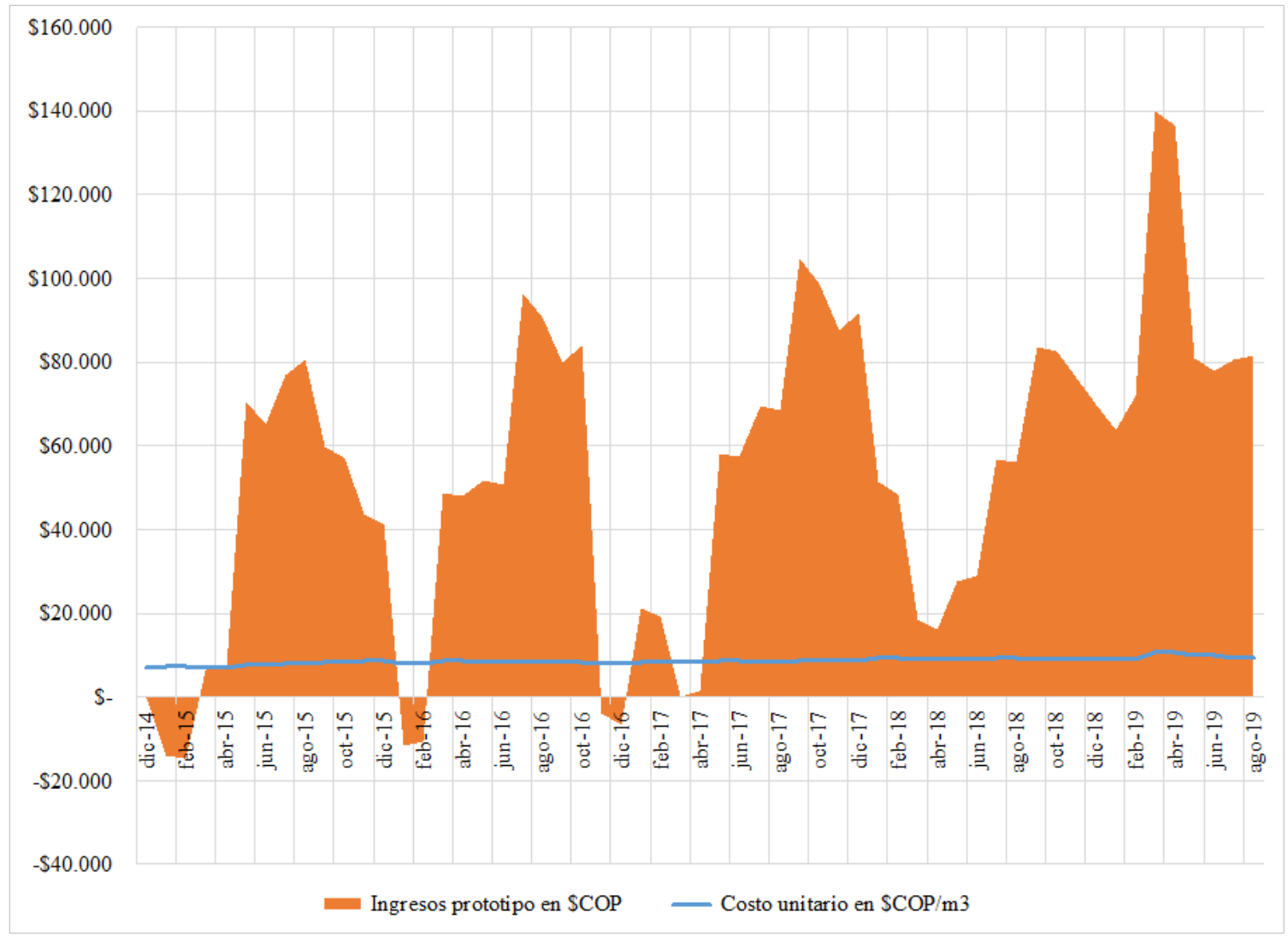

Figura 7. Ingresos del prototipo sistema inteligente en cascada para la cosecha de agua lluvia (SICCALL), por agua potable sustituida

Fuente: elaboración propia.

periodo de estudio; b) la EAB podría haber generado un programa de implementación de SCALL eficientes, para el abatimiento de GEI y participado en los mercados de carbono a través de los CRE, organizado bajo los mecanismos del protocolo de Kioto, con ello habría obtenido el 0,03\% de los ingresos dinerarios del prototipo. Con este programa, la EAB habría percibido un ahorro en su insumo energético que representa 1,64\% del total de ingresos dinerarios arrojados por el prototipo; c) el Distrito Capital de Bogotá, al impulsar como política el desarrollo del programa de SCALL eficientes alejaría la necesidad de ampliación de la capacidad del acueducto de Bogotá para garantizar la seguridad y confiabilidad del suministro de agua y energía. Además, considerando una penetración de SCALL eficientes, en un $30 \%$ de las viviendas NPH (no propiedad horizontal) de Bogotá (DANE, 2020), el Distrito Capital contabilizaría un ahorro energético de 3092,49 MWh y abatiría 977,28 $\mathrm{tCO}_{2}$ eq durante un periodo de 60 meses de implementación del programa. 
Prototipo de cosecha inteligente de agua lluvia para mejorar la eficiencia energética residencial en Bogotá Ramírez-Escobar., C.A. y Buriticá-Arboleda., C.I.

Tabla 5. Resultados de efectos económicos y de otros recursos en el periodo 2015-2019

\begin{tabular}{|c|c|c|}
\hline $\begin{array}{l}\text { Total ingresos del usuario del } \\
\text { prototipo por sustitución de } \\
\text { agua potable (COP) }\end{array}$ & $\begin{array}{l}\text { Total ingresos de EAB por } \\
\text { abatimiento de GEI en COP } \\
\text { (calculado para créditos de } \\
\text { carbono (CER)) }\end{array}$ & $\begin{array}{l}\text { Total ingresos por ahorro } \\
\text { energético de EAB en COP } \\
\text { (calculado para la tarifa } \\
\text { oficial industrial) }\end{array}$ \\
\hline COP 3032640 & COP 1132 & COP 50526 \\
\hline $\begin{array}{l}\text { Volumen de agua potable } \\
\text { de la línea base } \mathrm{m}^{3}\end{array}$ & $\begin{array}{c}\text { Huella de carbono del } \\
\text { suministro de agua potable kg } \\
\text { CO2eq }\end{array}$ & $\begin{array}{l}\text { Energía embebida del } \\
\text { agua potable kWh }\end{array}$ \\
\hline 1314,4 & 94,5 & 390 \\
\hline $\begin{array}{l}\text { Volumen de agua potable } \\
\text { ahorrada por el prototipo } \mathrm{m}^{3}\end{array}$ & $\begin{array}{c}\text { Abatimiento de GEI del } \\
\text { prototipo } \mathrm{kg} \mathrm{CO}_{2} \mathrm{eq}\end{array}$ & $\begin{array}{c}\text { Ahorro energético del } \\
\text { prototipo kWh }\end{array}$ \\
\hline 338,4 & 25 & 99 \\
\hline $\begin{array}{l}\text { \% de sustitución del } \\
\text { agua potable }\end{array}$ & $\begin{array}{l}\text { \% de abatimiento de GEI en } \\
\mathrm{CO}_{2} \text { eq por el prototipo }\end{array}$ & $\begin{array}{c}\text { \% de energía embebida en el } \\
\text { agua potable ahorrada }\end{array}$ \\
\hline 26 & 27 & 25 \\
\hline
\end{tabular}

Fuente: elaboración propia.

\section{Conclusiones}

La lluvia como recurso distribuido puede ser valorado tanto como recurso hídrico y energético, y así constatar la gran potencialidad de este recurso en Bogotá. Las metodologías de estudio de la eficiencia energética son adaptables para evaluar el ahorro de recursos que poseen, en forma embebida, la energía de servicios energéticos como el agua potable. Para este caso de estudio, la comparación de las intensidades energéticas de las actividades de suministro y sustitución, fueron un indicador útil para desarrollar la evaluación comparativa de la eficiencia. El diseño tarifario del suministro de agua potable y alcantarillado, así como las restricciones de participación de la demanda en mercados de energía eléctrica, le impiden a los usuarios finales y a la sociedad en general la captura de los beneficios económicos derivados de la implementación de los sistemas de cosecha de aguas lluvias (SCALL). Sin embargo, el análisis del prototipo muestra la recuperación de la inversión a partir de los ahorros producidos por la sustitución de agua potable.

Los resultados iniciales obtenidos permiten inferir sobre los potenciales beneficios individuales, sociales y ambientales de la implementación de políticas conducentes a estimular esta modalidad de gestión de la demanda de agua, a través de recursos distribuidos de agua y energía como la lluvia. 
Esto debido a que las autoridades administrativas de la ciudad podrían adelantar programas promotores de la eficiencia de agua y energía a través de inversiones en SCALL eficientes, por parte de los usuarios y partes interesadas, de manera que los recursos distribuidos tanto de agua lluvia, sol, viento y otros, bajo una economía red o colaborativa, rindieran los efectos de escala de beneficios significativos para los individuos, la sociedad y el planeta, convirtiendo a estos programas en programas meritorios de la futura "Bogotá, ciudad inteligente y eficiente".

\section{References}

[Acosta Alarcón, Rodríguez y Kua, 2019] Acosta Alarcón, R., Rodríguez, J. P. y Kua, H. W. (2019). Voluntary management of residential water demand in low and middle-low income homes: a pilot study of Soacha (Colombia). Water, 11(2), 216. DOI https://doi.org/10.3390/w11020216 个Ver página 175

[Agatón, Ruiz y Sayago, 2016] Agatón, A. L., Ruiz, J. C. C. y Sayago, U. F. C. (2016). Review of the status of art collection and utilization of rain water in urban and airports. Tecnura, 20(50), 141-153.

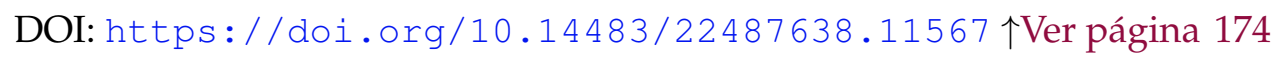

[Angrill et al., 2012] Angrill, S., Farreny, R., Gasol, C. M., Gabarrell, X., Viñolas, B., Josa, A. y Rieradevall, J. (2012). Environmental analysis of rainwater harvesting infrastructures in diffuse and compact urban models of Mediterranean climate. The International Journal of Life Cycle Assessment, 17(1), 25-42. DOI: https://doi.org/10.1007/s11367-011-0330-6个Ver página 177

[Bunyard y Herrera, 2012] Bunyard, P. y Herrera, F. (2012). El rol de la selva amazónica en la formación de las lluvias en Colombia. Intekhnia, 7(1), 27-36. ^Ver página 177

[Buriticá-Arboleda, Ramírez-Escobar y Álvarez-Bel, 2019] Buriticá-Arboleda, C. I., Ramírez-Escobar, C. A. y Álvarez-Bel, C. (2019). La seguridad de abastecimiento eléctrico en mercados liberalizados. Bogotá: Universidad Distrital Francisco José de Caldas. Recuperado de https://editorial. udistrital.edu.co/detalle.php?id=1166\&f=6 个Ver página 174

[Buriticá-Arboleda et al., 2020] Buriticá-Arboleda, C. I., Ramírez-Escobar, C., López Martínez, G., Moreno Ch., R., Martínez S, F. y Aldana M., F. (2020). Los recursos distribuidos de bioenergía en Colombia. Bogotá: Universidad Distrital. Recuperado de https://doctoradoingenieria. udistrital.edu.co/index.php/libroLosRecurDistriBioenerColom $\uparrow$ Ver página 178

[Carragher, Stewart y Beal, 2012] Carragher, B. J., Stewart, R. A. y Beal, C. D. (2012). Quantifying the influence of residential water appliance efficiency on average day diurnal demand patterns at an end use level: a precursor to optimized water service infrastructure planning. Resources, Conservation and Recycling, 62,81-90. DOI: https:// doi.org/10.1016/j.resconrec.2012.02.008 个Ver página 175 
[Climate-Data.org, 2019] Climate-Data.org (2019). Régimen bimodal de lluvias en Bogotá. Recuperado de https://es.climate-data.org/america-del-sur/colombia/bogota/ bogota-5115/\#climate-graph $\uparrow$ Ver página 177

[DANE, 2020] Departamento Administrativo Nacional de Estadística (DANE) (mayo 2020). Históricos Vivienda VIS y no VIS. Recuperado de https://www.dane.gov.co/ index.php/estadisticas-por-tema/construccion/vivienda-vis-y-no-vis/ vivienda-vis-y-no-vis $\uparrow$ Ver página 189

[EAB, 2014] Empresa de Acueducto de Bogotá (EAB) (2014). Informe de gases de efecto invernadero EABESP año 2014. Bogotá. $\uparrow$ Ver página 181

[EAB, 2015] Empresa de Acueducto de Bogotá (EAB) (2015). Informe de gases de efecto invernadero EABESP año 2015. Año comparativo No. 1: 2015 con año base: 2014. Bogotá. $\uparrow$ Ver página 181

[] Guide for conducting energy efficiency potential studies (No. 1219674; p. 1219674). National Action Plan for Energy Efficiency (2007). Guide for Conducting Energy Efficiency Potential Studies. Prepared by Philip Mosenthal and Jeffrey Loiter, Optimal Energy, Inc. www.epa.gov/ eeactionplan. $\uparrow$ Ver página

[García Lirios et al., 2013] García Lirios, C., Carreón Guillén, J., Hernández Valdés, J., Montero López Lena, M. y Bustos Aguayo, J. M. (2013). Actitudes, consumo de agua y sistema de tarifas del servicio de abastecimiento de agua potable. Polis. Revista Latinoamericana [En línea], 34. Recuperado de http://journals.openedition.org/polis/8933https://doi.org/10. 4067/S0718-65682013000100019个Ver página 175

[Goette, Leong y Qian, 2019] Goette, L., Leong, C. y Qian, N. (2019). Motivating household water conservation: a field experiment in Singapore. PLoS ONE, 14(3). DOI: https: // doi .org/10 . 1371 / journal.pone.0211891 $\uparrow$ Ver página 175

[ICONTEC, 2016] Instituto Colombiano de Normas Técnicas y Certificación (Icontec) (2016). Certificación Carbono Neutro EAA-ESP 2016-2019. Bogotá. $\uparrow$ Ver página 175

[IEA, 2014] International Energy Agency (IEA) (2014). Capturing the multiple benefits of energy efficiency: a guide to quantifying the value added. París. DOI: https://doi.org/10.1787/ 9789264220720 -en $\uparrow$ Ver página 178, 179

[Junca Salas, 1999] Junca Salas, J. C. (1999). Determinación del consumo básico de agua potable. Bogotá: Pontificia Universidad Javeriana. $\uparrow$ Ver página 182

[Ladino Tamayo, Martínez Rojas y Buriticá-Arboleda, 2018] Ladino Tamayo, A. F., Martínez Rojas, J. A. y Buriticá-Arboleda, C. I. (2018). Metodología APRENDYSAGE aplicada a los casos de estudio: 
Briceño (Boyacá) y Cajicá (Cundinamarca). Tecnura, 22(57), 13-31. DOI: https : / / do i . org/10 . $14483 / 22487638.13987 \uparrow$ Ver página 178

[Lizcano, Congote y Angarita, 2012] Lizcano, J. A., Congote, B. y Angarita, E. (2012). Exclusión del costo del agua en las tarifas de acueducto: La equívoca responsabilidad ambiental del servicio de acueducto y sus altas pérdidas. Tecnogestión: Una mirada al ambiente, 8(1), 24-30. DOI: https: //revistas.udistrital.edu.co/index.php/tecges/article/view/4380 个Ver página 174

[Makarieva y Gorshkov, 2007] Makarieva, A. M. y Gorshkov, V. G. (2007). Biotic pump of atmospheric moisture as driver of the hydrological cycle on land. Hydrology and Earth System Sciences, 11(2), 1013-1033. https://doi.org/10.5194/hess-11-1013-2007个Ver página 177

[Makarieva, Gorshkov y Li, 2009] Makarieva, A. M., Gorshkov, V. G. y Li, B.L. (2009). Precipitation on land versus distance from the ocean: evidence for a forest pump of atmospheric moisture. Ecological Complexity, 6(3), 302-307. https://doi.org/10.1016/j.ecocom.2008.11.004 个Ver página 177

[Manco Silva, Guerrero Erazo y Ocampo Cruz, 2012] Manco Silva, D. G., Guerrero Erazo, J. y Ocampo Cruz, A. M. (2012). Efficiency of residencial water consumption. Revista Ingenierías Universidad de Medellín, 11(21), 23-38. $\uparrow$ Ver página 175

[Mosenthal \& Loiter, 2007] Mosenthal, P., \& Loiter, J. (2007). Guide for Conducting Energy Efficiency Potential Studies. (p. 96) [Resource of the NAPEE]. National Action Plan for Energy Efficiency. https://archive.epa.gov/epa/statelocalclimate/ guide-conducting-energy-efficiency-potential-studies.html. $\uparrow$ Ver página 178, 179,180

[Pérez, Flores, González y Mota, 2019] Pérez, S. E. B., Flores, S. M., González, O. N. y Mota, R. de H. (2019). Implementación del método de escasez en la determinación de la huella hídrica en la zona costera de San Blas, México. Tecnura, 23(62), 45-54. DOI: https : / / doi . org/10 . 14483 / $22487638.15796 \uparrow$ Ver página 174

[Ramírez-Escobar, 2012] Ramírez-Escobar, C. A. (2012). Los precios del mercado mayorista de electricidad como expresión de la participación activa de la demanda: aplicación de la economía experimental. [Tesis de doctorado]. Universitat Politècnica de València, Valencia. Recuperado de https: / / riunet . upv.es/handle/10251/16806?show=full 个Ver página 174

[Ramírez-Escobar, 2007] Ramírez-Escobar, C. R. (2007). El uso racional de la energía desde la percepción de los mercados liberalizados de electricidad: respuesta de la demanda. Revista Clepsidra, 3(4), 27-35. Recuperado de http://revistas.fuac.edu.co/index.php/clepsidra/ article/view/264 $\uparrow$ Ver página 174 
[Retamal, Abeysuriya, Turner y White, 2009] Retamal, M., Abeysuriya, K., Turner, A. y White, S. (2009). The water-energy nexus: investigation into the energy implications of household rainwater systems. Sídney: Institute for Sustainable Futures. $\uparrow$ Ver página 174

[ScienceAtNASA, 2015] ScienceAtNASA (28 de abril de 2015). ScienceCasts: desert dust feeds Amazon forests [Archivo de video]. YouTube. Recuperado de. https : / www - youtube.com/watch? V=s7 IVGhTPQAY $\uparrow$ Ver página 177

[Sendanayake, 2010] Sendanayake, S. (2010). Development of an optimized integrated rainwater harvesting model for multistorey houses. [Tesis de doctorado]. University of Moratuwa, Moratuwa. Recuperado de http://dl.lib.mrt.ac.lk/handle/123/2033个Ver página 177

[Sendanayake, 2016] Sendanayake, S. (2016). Rainwater harvesting for urban living. Sri Lanka: South Asian Institute of Technology and Medicine. Recuperado de https://www.researchgate. net/publication/305380145_RAINWATER_HARVESTING_FOR_URBAN_LIVING $\uparrow$ Ver página 183

[Sharma, Begbie y Gardner, 2015] Sharma, A. K., Begbie, D. y Gardner, T. (2015). Rainwater tank systems for urban water supply. Londres: IWA Publishing. $\uparrow$ Ver página 176

[Stewart, Willis, Panuwatwanich y Sahin, 2013] Stewart, R. A., Willis, R. M., Panuwatwanich, K. y Sahin, O. (2013). Showering behavioral response to alarming visual display monitors: longitudinal mixed method study. Behaviour \& Information Technology, 32(7), 695-711. DOI: https : / / doi . org / $10.1080 / 0144929 x .2011 .577195 \uparrow$ Ver página 175

[Tijs et al., 2017] Tijs, M. S., Karremans, J. C., Veling, H., De Lange, M. A., Van Meegeren, P. y Lion, R. (2017). Saving water to save the environment: contrasting the effectiveness of environmental and monetary appeals in a residential water saving intervention. Social Influence, 12(2-3), 69-79. https://doi.org/10.1080/15534510.2017.1333967 ^Ver página 175

[Tjandraatmadja, Retamal, Umapathi y Hauber-Davidson, 2015] Tjandraatmadja, G., Retamal, M., Umapathi, S. y Hauber-Davidson, G. (2015). Understanding energy usage in rainwater tank systems through laboratory and household monitoring. En Rainwater tank systems for urban water supply (pp. 127-149). Londres: IWA Publishing. Recuperado de https://iwaponline.com/ ebooks/book-pdf/521261/wio9781780405360.pdf 个Ver página 176

[Ürge-Vorsatz et al., 2012] Ürge-Vorsatz, D., Eyre, N., Graham, P., Harvey, D., Hertwich, E., Jiang, Y., Kornevall, C., Majumdar, M., McMahon, J. E., Mirasgedis, S., Murakami, S., Novikova, A., Janda, K., Masera, O., McNeil, M., Petrichenko, K., Herrero, S. T. y Jochem, E. (2012). Energy end-use: buildings. En T. B. Johansson, N. Nakicenovic, A. Patwardhan y L. Gomez-Echeverri (eds.), Global Energy Assessment (GEA) (pp. 649-760). Cambridge: Cambridge University Press. 
https://www.cambridge.org/core/product/identifier/CB09780511793677A028/ type/book_part https://doi.org/10.1017/CB09780511793677.016 †er página 174

[US EPA, 2016] United States Environmental Protection Agency (US EPA) (12 de julio de 2016). WaterSense [Collections and Lists]. Washington. Recuperado de https://www.epa.gov/watersense 个Ver página 175

[Vieira et al., 2014] Vieira, A. S., Beal, C. D., Ghisi, E. y Stewart, R. A. (2014). Energy intensity of rainwater harvesting systems: a review. Renewable and Sustainable Energy Reviews, 34, 225-242. https://doi.org/10.1016/j.rser.2014.03.012个Ver página 175, 176 